\title{
Eficiência Hidráulica de Bocas de Lobo Situadas em Sarjetas de Greide Contínuo
}

\author{
Márcia Maria Lara Pinto Coelho, José Geraldo de Araújo Lima \\ Universidade Federal de Minas Gerais \\ Lara@ehr.ufmg.br; josegalima@uol.com.br
}

Recebido: 26/10/10 - revisado: 28/04/11 - aceito: 16/06/11

\section{RESUMO}

As bocas de lobo são estruturas hidráulicas importantes nos sistemas de drenagem urbana. Elas são responsáveis pela captação das águas que escoam nas sarjetas. Na maioria das cidades brasileiras, o dimensionamento dessas estruturas é fundamentado nos resultados de uma pesquisa cujo modelo físico difere em muitos detalhes daqueles utilizados atualmente em nossas vias públicas. Por essa razão, o presente trabalho apresenta os resultados obtidos com um modelo reduzido que procura reproduzir um pequeno segmento de rua, em greide contínuo, equipado com sarjeta e boca de lobo, à semelhança dos padrões adotados nos sistemas de drenagem de Belo Horizonte (MG). Com os testes realizados foi possível atribuir às bocas de lobo uma lei de captação para cada tipo testado. Como síntese dos principais resultados, destaca-se: as bocas de lobo apresentam maior eficiência hidráulica para as menores declividades longitudinais nas sarjetas; a boca de lobo simples, dotada de uma abertura na guia somente, apresenta as menores eficiências, enquanto a boca de lobo, dotada de grelha, abertura na guia e depressão na sarjeta, apresenta a maior eficiência hidráulica, podendo alcançar o valor de $100 \%$, para declividades longitudinais das sarjetas inferiores a 6\%; a contribuição da abertura na guia ou da depressão, numa boca de lobo combinada com depressão é reduzida, inferior a 8\%. Os testes também indicaram um bom ajuste da equação de Manning para o cálculo da vazão escoada na sarjeta. Uma estimativa para a vazão captada é proposta a partir dos dados de vazão escoada na sarjeta e na eficiência hidráulica da boca de lobo

Palavras-chave: Drenagem, boca de lobo, sarjeta.

\section{INTRODUCÃO}

Nos sistemas de drenagem urbana, as bocas de lobo são responsáveis pela captação das águas que escoam pelas sarjetas, conectando esses dispositivos de drenagem superficial à rede de galerias subterrâneas. Assim, para se ter um projeto adequado do sistema de drenagem é imprescindível o domínio teórico e prático das partes constituintes desse sistema e, portanto, da boca de lobo, tema do presente trabalho.

Os tipos de bocas de lobo mais utilizados nos sistemas de drenagem das cidades brasileiras são:

- a boca de lobo simples, com uma abertura na guia, através da qual é captada a água da sarjeta;

- a boca de lobo com grelha, constituída de uma abertura na sarjeta, coberta por uma grelha de barras metálicas ou de concreto;
- a boca de lobo combinada ou mista, correspondente a associação da boca de lobo simples com a de grelha.

A Figura 1 ilustra esses casos e mostra também que estas podem estar associadas ou não a depressões na sarjeta, utilizadas para concentrar a vazão captada.

O funcionamento das bocas de lobo depende do tipo adotado, da sua disposição na sarjeta e das condições hidráulicas desta.

A área livre para captação das águas pluviais superficiais da boca de lobo exerce uma grande influência na sua capacidade. Quando esta área livre é dotada de grelha, essa capacidade dependerá também da dimensão, da forma e disposição das barras da grelha.

Quanto à influência da sarjeta, as variáveis desta que condicionam o funcionamento hidráulico da boca de lobo são: as declividades transversais e longitudinais, a geometria da seção transversal e as rugosidades da sarjeta e da superfície do pavimento sobre a qual parte da água escoa. 
Dependendo da topografia, a boca de lobo pode estar situada em ponto baixo, neste caso a vazão adentra a abertura livre pelos dois lados ligados à sarjeta, ou pode estar numa rua de greide contínuo, caso em que a boca de lobo recebe a vazão somente pela sarjeta de montante.

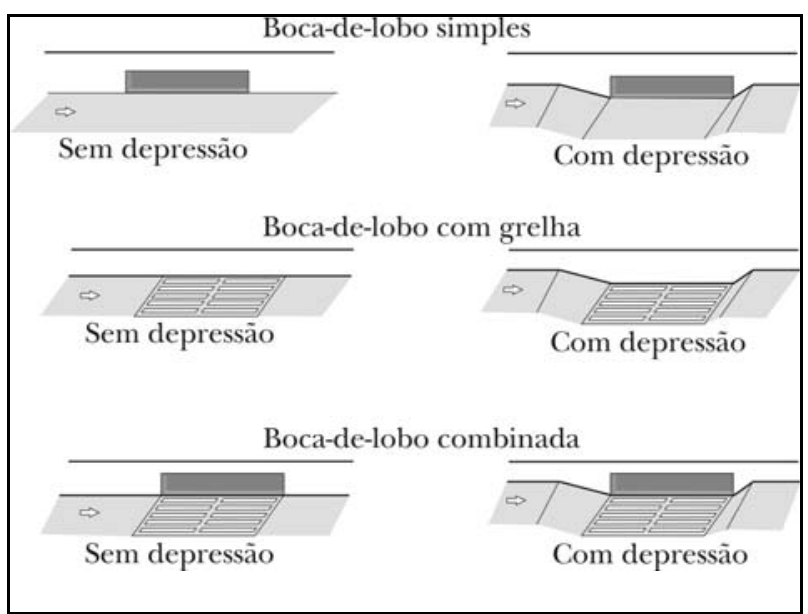

Figura 1 - Tipos de boca de lobo

Adaptado de DAEE/CETESB, 1980.

Por falta de pesquisas relacionadas ao desempenho hidráulico de alguns tipos de bocas de lobo, principalmente para aquelas dotadas de grelha, muitos projetos de drenagem são realizados com base nos resultados das pesquisas realizadas entre 1951 e 1954 pela Universidade Johns Hopkins, mesmo sabendo que as características anteriormente apontadas para a grelha e para sarjeta são diferentes. É objetivo desse trabalho, avaliar a capacidade de captação das águas superficiais de alguns tipos de bocas de lobo, por meio dos resultados de ensaios realizados num modelo físico que procura reproduzir um pequeno segmento de rua, em greide contínuo, equipado com sarjeta e boca de lobo, à semelhança dos padrões adotados nos sistemas de drenagem de Belo Horizonte (MG).

Espera-se com isto, contribuir na tarefa de aferir a eficiência hidráulica atribuída a estes dispositivos para determinadas condições de funcionamento.

Nas ruas de Belo Horizonte (MG), em geral, adota-se a boca de lobo combinada com grelha na sarjeta e abertura no meio fio, havendo na maioria das vezes uma depressão na sarjeta. A Figura 2 mostra este tipo de boca de lobo, empregado em trechos de greide contínuo, caso em que o fluxo atinge a boca de lobo apenas pelo lado de montante, onde o trecho de transição que forma a depressão é mais longo em relação ao lado de jusante. A grelha possui $0,99 \mathrm{~cm}$ de comprimento, por 47,5 cm de largura, com barras de $4 \mathrm{~cm}$, espaçadas de $4 \mathrm{~cm}$, dispostas conforme mostrado na Figura 3.

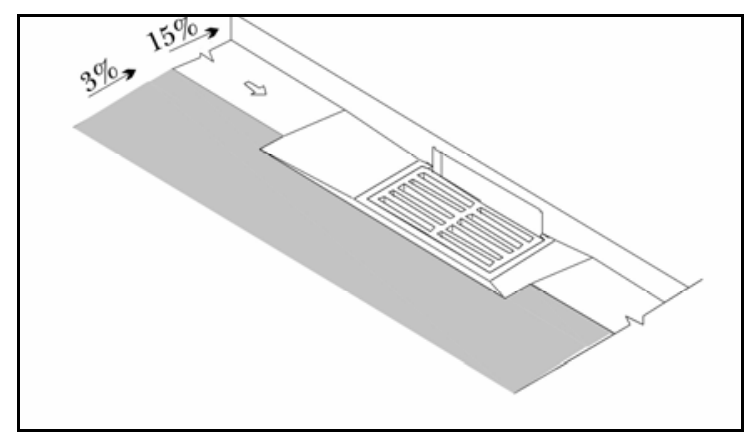

Figura 2 - Boca de lobo padrão SUDECAP

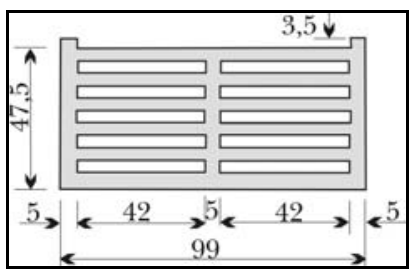

Figura 3 - Grelha padrão SUDECAP (dimensões em cm)

O tipo de boca de lobo mostrado é instalado em uma sarjeta padronizada como Tipo B pela SUDECAP (Superintendência de Desenvolvimento da Capital) com inclinação transversal de $15 \%$. Este padrão é recomendado para vias com declividades longitudinais iguais ou superiores a $0,5 \%$ e inferiores a $16 \%$, situação freqüente na maioria das vias urbanas. Por essa razão, esse tipo de sarjeta foi o adotado no presente trabalho.

\section{REVISÃO DA LITERATURA}

Apesar da importância da boca de lobo na dinâmica do funcionamento da microdrenagem, os estudos e pesquisas realizados sobre o tema, ainda não contemplam satisfatoriamente todas as situações de operação dessa estrutura hidráulica. Eastwood (1946), citado por Souza (1986) desenvolveu aquele que é considerado o mais antigo trabalho sobre 
boca de lobo. Posteriormente, um grupo de pesquisadores da Universidade Johns Hopkins (Benton, Geyer, Goodell, Li e Sorteberg), desenvolveu uma série de pesquisas para investigar os problemas decorrentes da drenagem de águas pluviais urbanas, em especial o comportamento hidráulico dos diversos tipos de boca de lobo. Estas pesquisas, ainda hoje, são referências para o dimensionamento dessa estrutura hidráulica. Algumas das observações importantes citadas nessas pesquisas são destacadas a seguir:

- A boca de lobo com grelha, constituída de barras transversais, em sarjetas sem depressão apresentou baixa eficiência de captação (Li, Geyer e Benton, 1951).

- A eficiência hidráulica para a boca de lobo simples com depressão foi $80 \%$, aproximadamente. Ao retirar a depressão, a eficiência hidráulica foi reduzida para quase $60 \%$, na maioria dos casos testados (Li, Geyer e Sorteberg, 1951).

- Li et al. (1954) propôs um tipo de boca de lobo não convencional, dotado de um conjunto de defletores (ranhuras) na sarjeta dispostos em espaços regulares ao longo da abertura na guia. Estes defletores permitiram aumentar a capacidade da boca de lobo, principalmente para as declividades longitudinais mais elevadas, uma característica contrária às mostradas pelos outros tipos de boca de lobo (Li, Goodell e Geyer, 1954).

- O número de barras transversais na grelha reduziu a eficiência da boca de lobo $(\mathrm{Li}$, Goodell e Geyer, 1954).

Souza (1986) realizou um estudo experimental para a determinação da eficiência de uma boca de lobo simples (com e sem depressão), usando dois modelos físicos, sendo um na escala 1:3 e o outro na escala 1:1. Em ambos os modelos, as declividades longitudinais estiveram entre 5 e $14 \%$ e as vazões dos testes situadas na faixa de eficiência entre 50 e $100 \%$. Neste estudo concluiu-se que, para o modelo estudado, a boca de lobo com depressão na sarjeta é em torno de $36 \%$ mais eficiente do que a boca de lobo sem depressão. Para uma vazão fixada, ambos os tipos de boca de lobo perderam eficiência com o aumento da declividade longitudinal. A partir dos dados experimentais foi proposto um modelo matemático para determinar a vazão captada pela boca de lobo simples, com e sem depressão.
O trabalho de Souza (1986) relata ainda outras experiências nessa área, destacando: os resultados experimentais de Yong (1965), obtidos com um modelo hidráulico reduzido (escala 1:4) para boca de lobo simples; experiências de campo e em modelos reduzidos executadas por Zwamborn (1966), obtendo confirmação da fórmula originária dos estudos realizados na Universidade Johns Hopkins; estudos experimentais de Russam (1969) a respeito da eficiência hidráulica de bocas de lobo.

Mais recentemente, Dalfré e Genovez (2004) testaram em laboratório um modelo de boca de lobo simples, sem depressão, mas com um rasgo adicional na guia. Como resultado, obteve-se um considerável acréscimo na eficiência hidráulica da boca de lobo, encontrando na lei de captação do modelo ensaiado coeficientes de descarga até duas vezes superiores aos coeficientes citados em outras pesquisas feitas com bocas de lobo simples e sem rasgo adicional na guia.

Kriguer e Contreras (2004) apoiando-se num modelo em escala real (1:1) fizeram experiências para a verificação da eficiência hidráulica de uma boca de lobo com grelha metálica, constituída de barras transversais e longitudinais, padrão utilizado em algumas cidades do Chile, mas que diferem bastante dos padrões utilizados na maioria das cidades brasileiras.

Cardoso et al. (2004) apoiados num modelo em escala 1:3 realizaram experiência com boca de lobo com defletores instalados defronte a abertura na guia e no sentido perpendicular ao fluxo da sarjeta. Este tipo de boca de lobo apresentou em sua lei de captação um coeficiente de descarga superior a duas vezes o coeficiente encontrado em outras pesquisas feitas com bocas de lobo sem defletores na sarjeta, defronte a abertura na guia.

Para a determinação da capacidade das bocas de lobo situadas em sarjetas de greide contínuo, no padrão adotado nesta pesquisa, a SUDECAP (2004) recomenda a utilização dos estudos realizados pela Universidade Johns Hopkins, para bocas de lobo combinadas, situadas na depressão. Os valores de referência são 102 l/s, para a declividade de $5 \%$ e $128 \mathrm{l} / \mathrm{s}$ para a declividade de $8 \%$. Para declividades maiores é recomendada a adoção de bocas de lobo dupla.

Lima (2007) e Lima \& Coelho (2007) apresentaram um estudo detalhado das bocas de lobo situadas em sarjetas de greide contínuo. Um modelo reduzido em escala 1:3 foi utilizado para verificar o comportamento hidráulico dessas bocas de lobo. Buscando melhorar a eficiência destas bocas de lobo, vários tipos de grelha foram pesquisados. A 
grelha com barra transversal posicionada sob as barras longitudinais proporcionou aumento de $10 \%$, aproximadamente, na capacidade de captação das bocas de lobo. Os resultados experimentais destas pesquisas constituem a base para o trabalho ora apresentado.

\section{METODOLOGIA}

A eficiência hidráulica de uma boca de lobo faz-se pela determinação da relação entre a vazão captada e a vazão afluente da sarjeta. Para as bocas de lobo situadas em segmentos de greides contínuos, a vazão captada depende da altura da lâmina de água a montante desta, que por sua vez depende das características geométricas da sarjeta, bem como do comprimento, da largura e das demais variáveis geométricas da boca de lobo. Assim, a vazão captada pela boca de lobo com grelha e abertura na guia pode ser dada pela relação funcional:

$\varphi_{1}\left(Q, Q_{0}, y_{0}, L, A_{u}, e, \theta, I, n, G\right)=0$

em que,

Q: $\quad$ vazão captada pela boca de lobo $\left(\mathrm{m}^{3} / \mathrm{s}\right)$;

$\mathrm{Q}_{0}$ : $\quad$ vazão que aflui pela sarjeta $\left(\mathrm{m}^{3} / \mathrm{s}\right)$;

$\mathrm{y}_{0}$ : altura da lâmina d'água a montante da boca de lobo $(\mathrm{m})$;

L: comprimento da abertura na guia ou na grelha $(\mathrm{m})$;

$\mathrm{A}_{\mathrm{u}}$ : área útil das aberturas na grelha $\left(\mathrm{m}^{2}\right)$;

e : $\quad$ largura da abertura na grelha $(\mathrm{m})$;

$\theta$ : $\quad$ ângulo do plano da sarjeta com a vertical;

I : $\quad$ declividade longitudinal da sarjeta $(\mathrm{m} / \mathrm{m})$;

$\mathrm{n}$ : coeficiente de rugosidade da equação de Manning da sarjeta;

G : parâmetro adimensional que descreve a geometria da sarjeta nas proximidades da boca de lobo.

A utilização da análise dimensional na relação (1) permite deduzir para uma dada geometria da sarjeta nas proximidades da boca de lobo as relações adimensionais expressas a seguir:

$$
\frac{\mathrm{Q}}{\mathrm{Q}_{0}}=\varphi_{2}\left(\frac{\mathrm{L}}{\mathrm{y}_{0}}, \mathrm{I}, \mathrm{n}\right)
$$

Medindo-se as grandezas envolvidas nesta equação a função $q$ : pode ser determinada, para um modelo de boca de lobo e sarjeta definido. Entretanto, devido à grande dificuldade em se medir e controlar essas grandezas numa situação real de campo é recomendável a utilização de modelo físico.

Para tanto, foi construído um modelo físico, na escala 1:3, no Centro de Pesquisas Hidráulicas da UFMG. Este modelo se baseou num protótipo correspondente a um trecho de rua com sarjeta padrão $\mathrm{B}$ da SUDECAP, com 6,0 m de comprimento por $0,75 \mathrm{~m}$ de largura. A sarjeta possui duas declividades transversais, a primeira, adjacente ao meio fio, de largura $\mathrm{W}$ igual a $0,17 \mathrm{~m}$ no modelo, na faixa de concreto, com $15 \%$ de declividade transversal e a outra, com $3 \%$ de declividade transversal, refere-se à faixa da pista atingida pela lâmina d'água, conforme ilustram as Figura 2 e 4 . Conforme SUDECAP (2004) a largura de alagamento total(T) deve ser igual ou inferior a 1,67 m no protótipo, equivalente a $0,56 \mathrm{~m}$ no modelo. Segundo a mesma fonte, admite-se coeficiente de Manning $n$ de 0,015 para o cálculo das vazões.

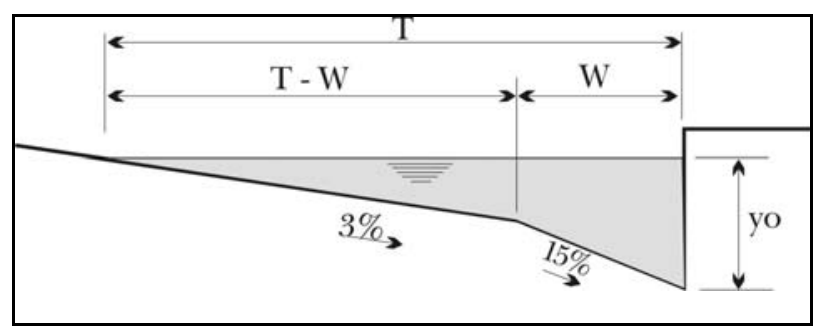

Figura 4- Seção transversal sarjeta

Próximo ao fim da sarjeta de greide contínuo há uma caixa adaptável aos cinco tipos de boca de lobo ensaiados, cuja denominação e características relacionadas à depressão na sarjeta, abertura na guia estão relacionadas na Tabela 1 , apresentada a seguir:

Tabela 1 - Tipos de boca de lobo ensaiados e suas características

\begin{tabular}{c|c|c|c}
\hline \multirow{2}{*}{ Denominação } & \multicolumn{3}{|c}{ Características } \\
\cline { 2 - 4 } & $\begin{array}{c}\text { Depressão } \\
\text { na sarjeta }\end{array}$ & $\begin{array}{c}\text { Abertura } \\
\text { na guia }\end{array}$ & Grelha \\
\hline BL-1 & não & sim & não \\
BL-2 & não & sim & sim \\
BL-3 & não & não & sim \\
BL-4 & sim & sim & sim \\
BL-5 & sim & não & sim \\
\hline
\end{tabular}


A grelha utilizada na pesquisa é semelhante àquela mostrada na Figura 3, sendo a relação geométrica entre o protótipo e o modelo de 1:3.

A Figura 5 mostra um esquema do arranjo do modelo conectado ao módulo de bombeamento, encarregado da recirculação e ao canal de saída, dotado de um vertedouro triangular, para medida da vazão captada.

As bocas de lobo foram ensaiadas para as declividades longitudinais de 0,5\%,5\%,8\%, $11 \%$ e $14 \%$ e valores de vazões afluentes definidos de acordo com a lâmina de água na sarjeta, sendo os valores máximos correspondentes às larguras máximas admissíveis na sarjeta, igual a $0,56 \mathrm{~m}$ no modelo. A partir desse valor, as vazões foram reduzidas gradualmente até atingir um valor de eficiência de $100 \%$, no qual toda a vazão escoada era captada pela boca de lobo.

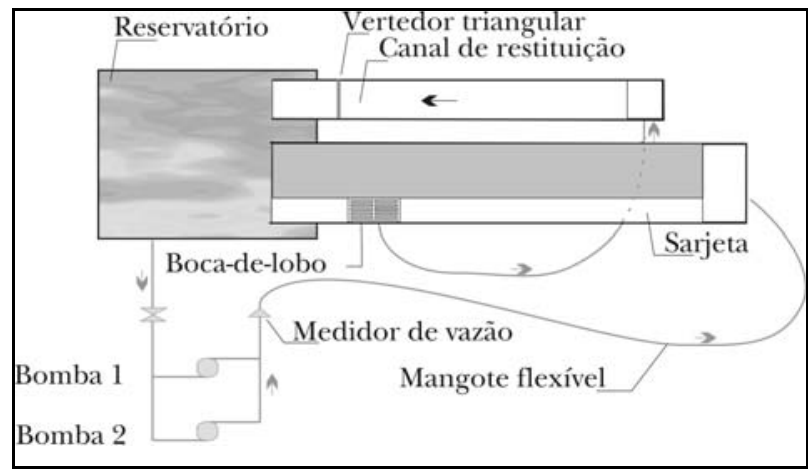

Figura 5 -Arranjo do modelo

As Figuras 6 e 7, apresentadas a seguir, mostram duas fotografias referentes às etapas de construção do modelo físico, respectivamente, após a conclusão dos trabalhos de marcenaria e a outra, com o modelo revestido com fibra de vidro e resina e também pintado com cores que caracterizam a sarjeta e a pista.

Nos modelos de escoamentos livres as forças preponderantes são as gravitacionais, viscosas e inerciais. Contudo, se os escoamentos no modelo e no protótipo forem turbulentos rugosos, ainda que os números de Reynolds sejam diferentes pode haver uma relaxação das forças viscosas. Isso facilita a análise do escoamento, visto que as forças atuantes serão predominantemente as forças de inércia e gravitacional.

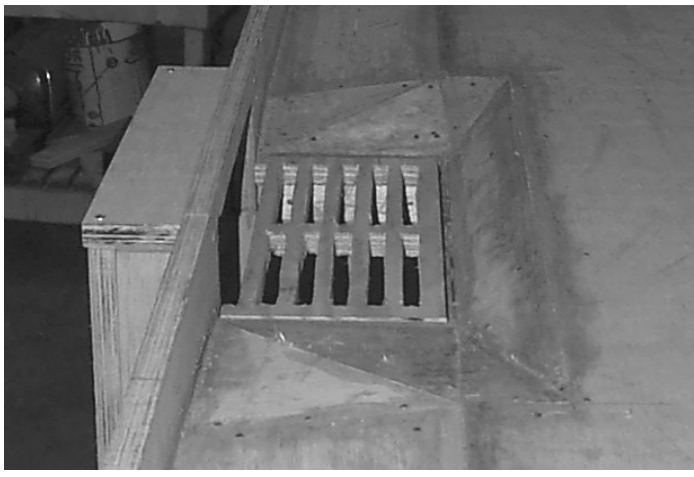

Figura 6 - Vista da boca de lobo com grelha e abertura na guia, com depressão, após a conclusão dos serviços de marcenaria.

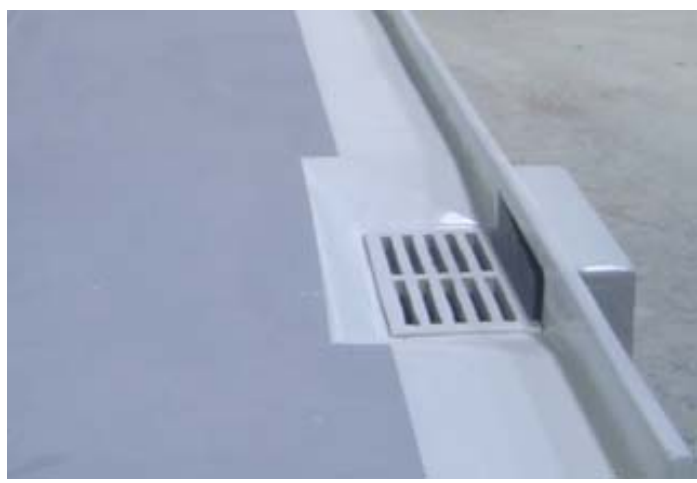

Figura 7 - Vista do modelo da faixa de rua dotado de sarjeta, boca de lobo com grelha e abertura na guia, revestido de fibra de vidro, resina e pintura.

Para averiguar a possibilidade dessa relaxação foi investigado o tipo de escoamento predominante nas sarjetas de duas ruas de Belo Horizonte. A primeira revestida com asfalto, declividade transversal de $10 \%$ e longitudinal de $14 \%$ e a segunda em paralelepípedo e declividade longitudinal de $9 \%$. O levantamento contou com o apoio de membros do Corpo de Bombeiros que abriram os hidrantes existentes nos locais da pesquisa e determinaram com o emprego de um tubo Pitot as vazões lançadas nas sarjetas. Foram medidas as lâminas d'água para os três valores de vazão lançados em cada rua, sendo as características geométricas das sarjetas e as declividades longitudinais das ruas definidas topograficamente. Em seguida foram calculados os parâmetros hidráulicos relativos ao escoamento na sarjeta, com a hipótese de escoamento uniforme, obtendo: velocidade média $(U)$, raio hidráulico $(R h)$, número de Reynolds $(R e)$ e o fator de atrito da equação Univer- 
sal de perda de carga $f$, conforme consta da Tabela 2. Vale destacar que o valor de $f$ foi calculado pela equação Universal de perda de carga, considerando a perda de carga unitária igual a declividade da sarjeta. Os valores de $\mathrm{f}^{1 / 2}$ versus Re. $\mathrm{f}^{1 / 2}$ quando plotados no ábaco de Rouse localizaram-se na zona do escoamento turbulento rugoso. Desta maneira foi possível concluir que as forças viscosas não são preponderantes nesse tipo de escoamento e que o modelo e o protótipo podem ser considerados semelhantes dinâmicamente, se estes tiverem o mesmo número de Froude, sendo este dado pela seguinte relação:

$$
\mathrm{Fr}=\frac{\mathrm{U}}{\sqrt{\mathrm{gy}}}
$$

Tabela 2 - Parâmetros para a definição do tipo de escoamento ocorrido na sarjeta

\begin{tabular}{lllllll}
\hline \multirow{2}{*}{ Rua } & $\mathrm{Q}_{0}$ & $\mathrm{y}_{0}$ & $\mathrm{U}$ & $\mathrm{Rh}$ & $\mathrm{Re}$ & $\mathrm{f}$ \\
& $\mathrm{l} / \mathrm{s}$ & $\mathrm{m}$ & $\mathrm{m} / \mathrm{s}$ & $\mathrm{m}$ & & \\
\hline \multirow{3}{*}{$\mathrm{I}$} & 24 & 0,45 & 2,40 & 0,0204 & 182692 & 0,040 \\
& 31 & 0,55 & 2,10 & 0,0249 & 192971 & 0,065 \\
& 44 & 0,66 & 2,00 & 0,0299 & 227419 & 0,081 \\
\hline \multirow{4}{*}{ II } & 28 & 0,70 & 1,26 & 0,0301 & 143007 & 0,209 \\
& 46 & 0,75 & 1,96 & 0,0300 & 221451 & 0,085 \\
& 50 & 0,83 & 1,72 & 0,0335 & 216539 & 0,125 \\
\hline
\end{tabular}

Considerando a utilização de água nas mesmas condições de temperatura e pressão, tanto no modelo quanto no protótipo e o critério de semelhança dinâmica de Froude, as grandezas físicas envolvidas no fenômeno se relacionam com a razão de escala $L_{r}$, por meio das equações apresentadas a seguir, onde os índices $\mathrm{m}$ e $\mathrm{p}$ se referem, respectivamente, ao modelo e ao protótipo.

comprimento: $\quad L_{r}=\frac{L_{m}}{L_{p}}=\frac{R h_{m}}{R h_{p}}$

velocidade: $\quad \mathrm{U}_{\mathrm{r}}=\frac{\mathrm{U}_{\mathrm{m}}}{\mathrm{U}_{\mathrm{p}}}=\mathrm{L}_{\mathrm{r}}^{1 / 2}$

vazão: $\quad Q_{r}=\frac{Q_{m}}{Q_{p}}=L_{r}^{5 / 2}$

Outro tipo de esforço comum nos modelos hidráulicos reduzidos é devido à tensão superficial. Todavia, esse efeito pode ser desprezível ao se ado- tar modelos que não possibilitem lâminas d'água inferiores a $1,0 \mathrm{~cm}$.

\section{RESULTADOS}

Na seqüência serão apresentados os resultados obtidos nos ensaios realizados. Primeiramente serão tratados os dados referentes à calibração da rugosidade do modelo. Serão também analisados os dados referentes à capacidade da sarjeta adotada. Finalmente, serão apresentados e analisados os gráficos com os resultados dos testes realizados com os diversos tipos de bocas de lobo pesquisados.

\section{Calibração da rugosidade do modelo}

O modelo físico, revestido com fibra de vidro, resina e pintura, tal como mostrado na Figura 7, quando posto em operação, apresentou escoamento do tipo turbulento liso. Visando adequá-lo às condições de escoamento turbulento rugoso, buscou-se aumentar a rugosidade da superfície do modelo, ajustando assim às condições verificadas nos testes preliminares de campo. A princípio fez-se o seu revestimento com uma camada de $4 \mathrm{~mm}$ de argamassa, sobre a qual foi espalhada e prensada areia graduada por peneiramento, com $100 \%$ dos grãos passando na peneira 10 e $100 \%$ retidos na peneira 16 . O coeficiente de rugosidade de Manning para o novo revestimento foi da ordem de 0,009, não permitindo ainda o escoamento turbulento rugoso.

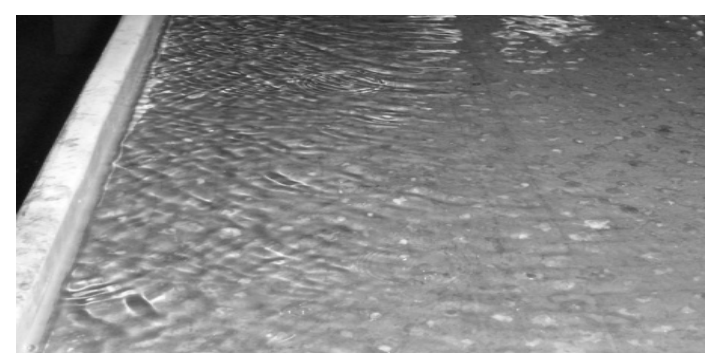

Figura 8- Aspectos do escoamento após o revestimento final da superfície do modelo

Como nova medida para aumento da rugosidade optou-se pela colagem de pedras sobre a argamassa assentada na fase anterior, usando uma densidade média de 32 pedras a cada dez centíme- 
tros quadrados. Neste revestimento, feito manualmente, foram usadas pedras de aquário com diâmetro aproximado de $4 \mathrm{~mm}$. As alterações assim processadas na superfície permitiram escoamento turbulento rugoso, com coeficiente de Manning de 0,0118, com aspecto ilustrado na Figura 8. Embora este valor ainda estivesse abaixo do valor do protótipo $(n=0,015)$, optou-se por trabalhar com o modelo distorcido e fazer as devidas compensações nos resultados finais.

\section{Capacidade da sarjeta}

Conforme descrito anteriormente, os critérios desenvolvidos para a determinação da capacidade dos diversos tipos de boca de lobo baseiam-se nos parâmetros relativos ao escoamento na sarjeta, tais como altura da lâmina d'água no meio fio, $\mathrm{y}_{\mathrm{o}}$, velocidade e vazão, $U_{o}$ e $Q_{0}$. A equação de Manning para escoamento uniforme em canais, com escoamento turbulento rugoso é a maneira mais usual de obter esses parâmetros.

A Figura 9 mostra um gráfico de vazão versus declividade para o modelo, destacando os resultados experimentais para as declividades longitudinais de $5 \%, 8 \%, 11 \%$ e $14 \%$, bem como as curvas obtidas por meio das equações de Manning e Izzard, para n=0,0118, considerando a faixa de alagamento de $T$ $=0,56 \mathrm{~m}$. As equações (7) e (8) mostradas a seguir são resultantes das formulações de Manning e Izzard, respectivamente:

$\mathrm{Q}_{0}=0,0258 . \mathrm{I}^{0.5}$

$\mathrm{Q}_{0}=0,0375 . \mathrm{I}^{0.5}$

Sendo $Q_{0}$ a vazão que escoa na sarjeta $\mathrm{em}^{3} / \mathrm{s}$ e I a declividade longitudinal desta, em $\mathrm{m} / \mathrm{m}$.

Para empregar a equação de Izzard no presente caso, a contribuição da face da guia para cálculo do perímetro molhado foi desprezada, conduzindo, assim, a resultados mais elevados para a vazão, conforme se pode constatar na Figura 9.

Tendo em vista o bom ajuste da equação de Manning aos dados experimentais, com erro médio relativo da ordem de $9 \%$, a mesma foi utilizada para o protótipo, considerando a distorção havida entre os coeficientes de rugosidade $\mathrm{n}$ do modelo e do protótipo, que são 0,0118 e 0,015 , respectivamente. Desta maneira, obtém a capacidade da sarjeta em função da declividade para o protótipo, cuja equação para $\mathrm{T}=1,67 m$ é a seguinte:

$Q_{0}=0,380 \cdot I^{0.5}$
Sendo $Q_{0}$ a vazão que escoa na sarjeta $\mathrm{em}^{3} / \mathrm{s}$ e I a declividade longitudinal desta, em $\mathrm{m} / \mathrm{m}$.

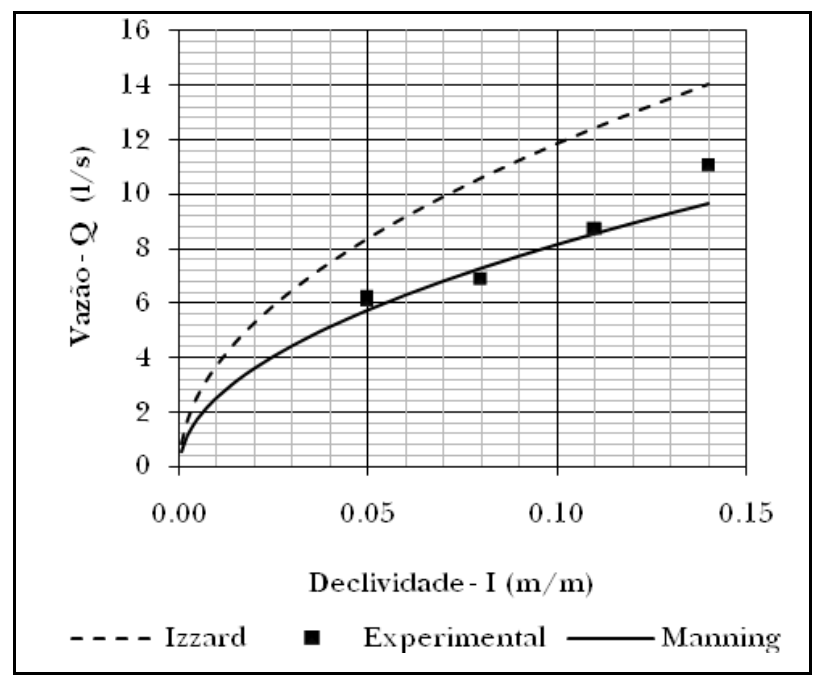

Figura 9 - Vazão versus declividade para o modelo

\section{Capacidade das bocas de lobo}

A capacidade de captação das bocas de lobo com entrada pela guia BL-1 pode ser comparada a de um vertedor de entrada lateral, cuja lei de captação é definida por:

$\mathrm{Q} / \mathrm{L}=\mathrm{kyo}^{1,5}$

em que,

Q: vazão captada na boca de lobo $\mathrm{em}^{3} / \mathrm{s}$;

L: largura da abertura na guia em $\mathrm{m}$;

yo: profundidade imediatamente a montante da boca de lobo em $\mathrm{m}$;

k: fator que depende do coeficiente de descarga e do ângulo da inclinação transversal da sarjeta, a ser determinado experimentalmente.

Os resultados dos testes para a boca de lobo BL-1 demonstraram que a vazão captada foi pouco afetada pela variação da declividade da sarjeta, conforme ilustra a Figura 10. Isso possibilitou a representação da vazão captada pela largura de abertura na guia $(\mathrm{Q} / \mathrm{L})$ por uma equação dependente somente da profundidade imediatamente a montante da boca de lobo (yo).

Para as demais bocas de lobo (BL-2, BL-3, BL-4 e BL-5), constituídas de grelha com ou sem 
depressão, o ajuste por meio da equação 10 não se mostrou satisfatório. A relação apresentada em (11), também dependente de yo ${ }^{1,5}$, pareceu mais adequada para estes casos, onde as constantes $\mathrm{k}_{1}$ e $\mathrm{k}_{2}$ foram determinadas experimentalmente, para cada tipo de boca de lobo, segundo as declividades longitudinais estabelecidas.

$\mathrm{Q} / \mathrm{L}=\mathrm{k}_{1} \mathrm{yo}^{1,5}-\mathrm{k}_{2}$

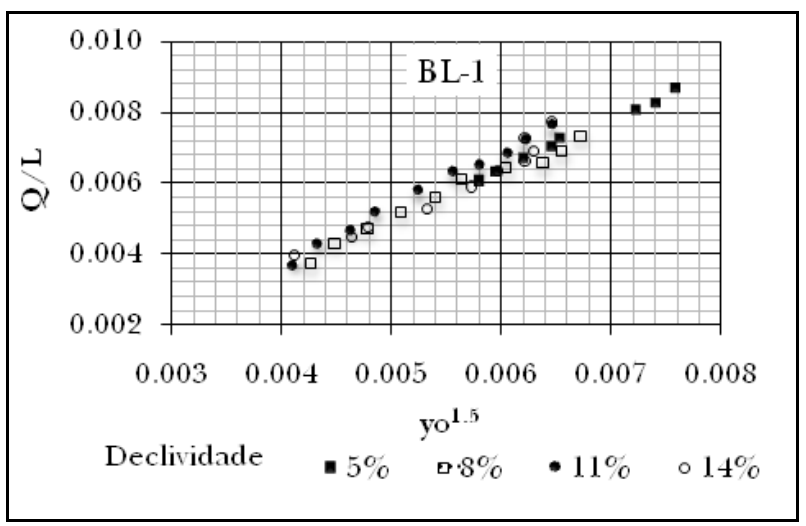

Figura 10 - Capacidade de captação da boca de lobo BL-1

A seguir são apresentados os gráficos dos resultados de $\mathrm{Q} / \mathrm{L}$ em função de yo ${ }^{1,5}$ para os testes realizados com as bocas de lobo BL-2, BL-3, BL-4 e BL-5, cujas características foram apresentadas na Tabela 1.

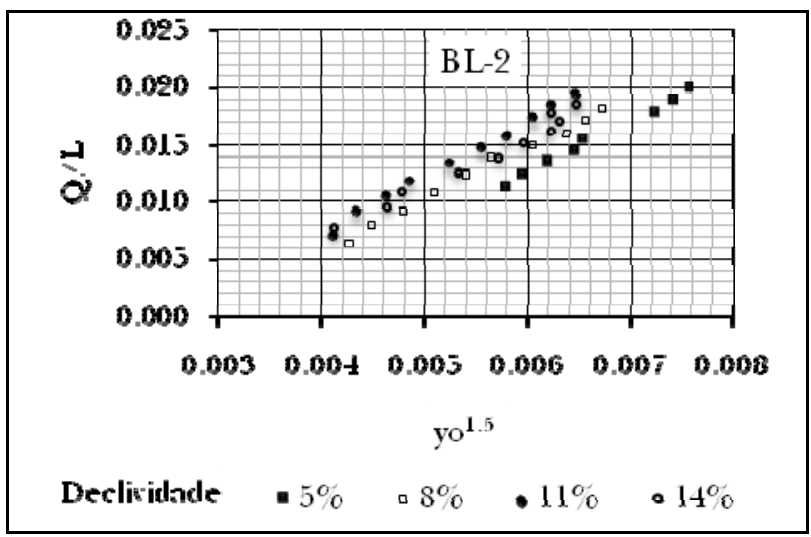

Figura 11 - Capacidade de captação da boca de lobo BL-2

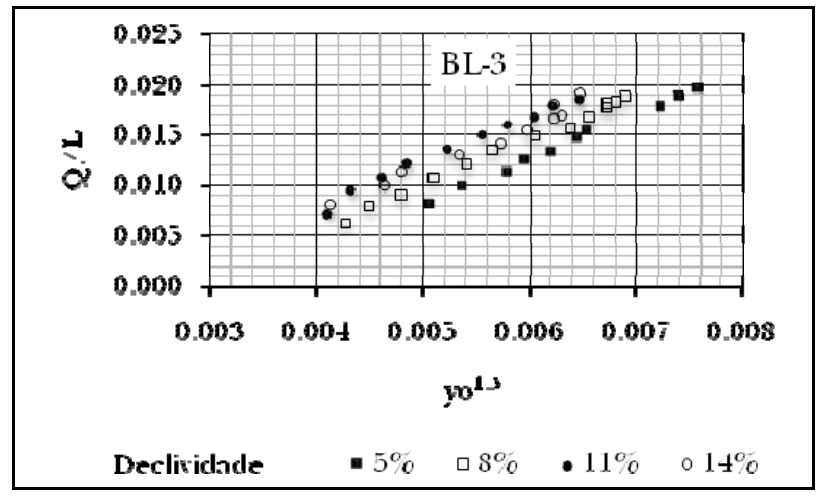

Figura 12 - Capacidade de captação da boca de lobo BL-3

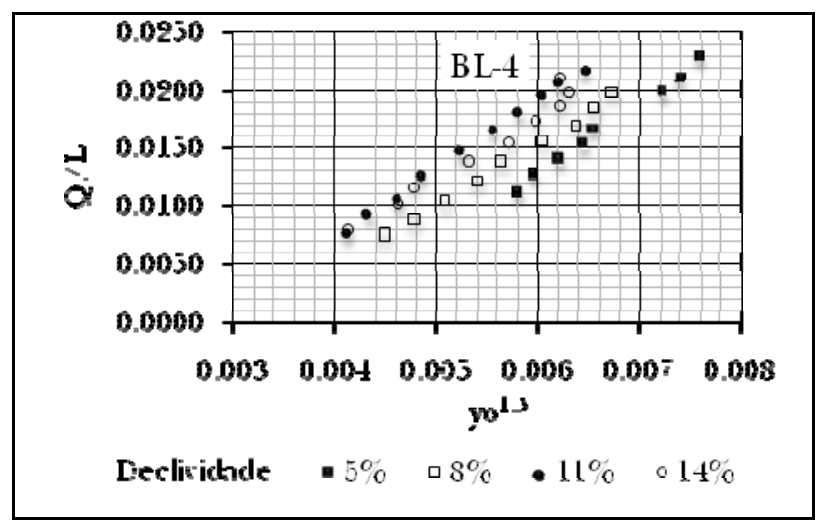

Figura 13 - Capacidade de captação da boca de lobo BL-4

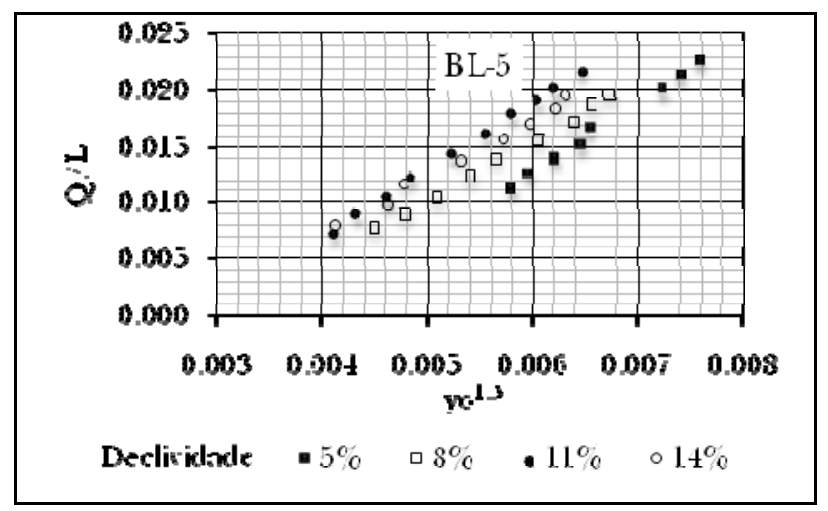

Figura 14 - Capacidade de captação da boca de lobo BL-5

As equações que relacionam as variáveis apresentadas nos gráficos mostrados nas Figuras 10 a 14 estão contidas na Tabela 3 . Os coeficientes de correlação $\left(\mathrm{R}^{2}\right)$ dessas equações variaram entre os valores de 0,92 a 1,00, indicando um bom ajuste da relação $\mathrm{Q} / \mathrm{L}$ com yo ${ }^{1,5}$. 
Tabela 3 - Vazão captada por comprimento de boca- de lobo - $Q / L,\left(\mathbf{m}^{3} / \mathbf{s . m}\right)$, em função de yo (m) e da declividade $I(\%)$

\begin{tabular}{l|l|l|l|l}
\hline $\begin{array}{l}\text { Tipo de } \\
\text { Boca de lobo }\end{array}$ & Q/L para & Q/L para & Q/L para & Q/L para \\
$\mathrm{I}=5 \%$ & $\mathrm{I}=8 \%$ & $\mathrm{I}==11 \%$ & $\mathrm{I}==14 \%$ \\
\hline BL-1 & $1,08 \mathrm{yo}^{1,5}$ & $1,08 \mathrm{yo}^{1,5}$ & $1,08 \mathrm{yo}^{1,5}$ & $1,08 \mathrm{yo}^{1,5}$ \\
BL-2 & $4,57 \mathrm{yo}^{1,5}-0,015$ & $4,58 \mathrm{yo}^{1,5}-0,013$ & $4,89 \mathrm{yo}^{1,5}-0,012$ & $4,41 \mathrm{yo}^{1,5}-0,011$ \\
BL-3 & $4,49 \mathrm{yo}^{1,5}-0,015$ & $4,56 \mathrm{yo}^{1,5}-0,013$ & $4,47 \mathrm{yo}^{1,5}-0,010$ & $4,44 \mathrm{yo}^{1,5}-0,010$ \\
BL-4 & $6,21 \mathrm{yo}^{1,5}-0,025$ & $5,43 \mathrm{yo}^{1,5}-0,017$ & $6,08 \mathrm{yo}^{1,5}-0,017$ & $5,61 \mathrm{yo}^{1,5}-0,016$ \\
BL-5 & $6,20 \mathrm{yo}^{1,5}-0,025$ & $5,39 \mathrm{yo}^{1,5}-0,017$ & $6,11 \mathrm{yo}^{1,5}-0,018$ & $5,21 \mathrm{yo}^{1,5}-0,014$ \\
\hline
\end{tabular}

Nos experimentos de Souza (1986) para uma boca de lobo do tipo BL-1 a relação entre $(\mathrm{Q} / \mathrm{L})$ e yo ${ }^{1,5}$ foi de 0,66 , enquanto no atual foi 1,08 . A justificativa para essa variação se deve, principalmente, a diferença entre as geometrias e rugosidades das sarjetas.

Conhecida a vazão captada no modelo para largura de alagamento máxima $(\mathrm{T}=0,56 \mathrm{~m}$, correspondente a yo=0,037 m), foi possível estimar, para cada tipo de boca de lobo ensaiada, a vazão captada no protótipo com $(\mathrm{T}=1,67 \mathrm{~m}$, yo $=0,11 \mathrm{~m}$,$) , relacio-$ nando as vazões pela razão de escala (ver equação 6). Com base nessas informações e na capacidade da sarjeta do protótipo(ver equação 9) foi calculada a eficiência hidráulica, (Q/Qo), em função da declividade, cujo gráfico destas variáveis estão ilustrados na Figura 15.

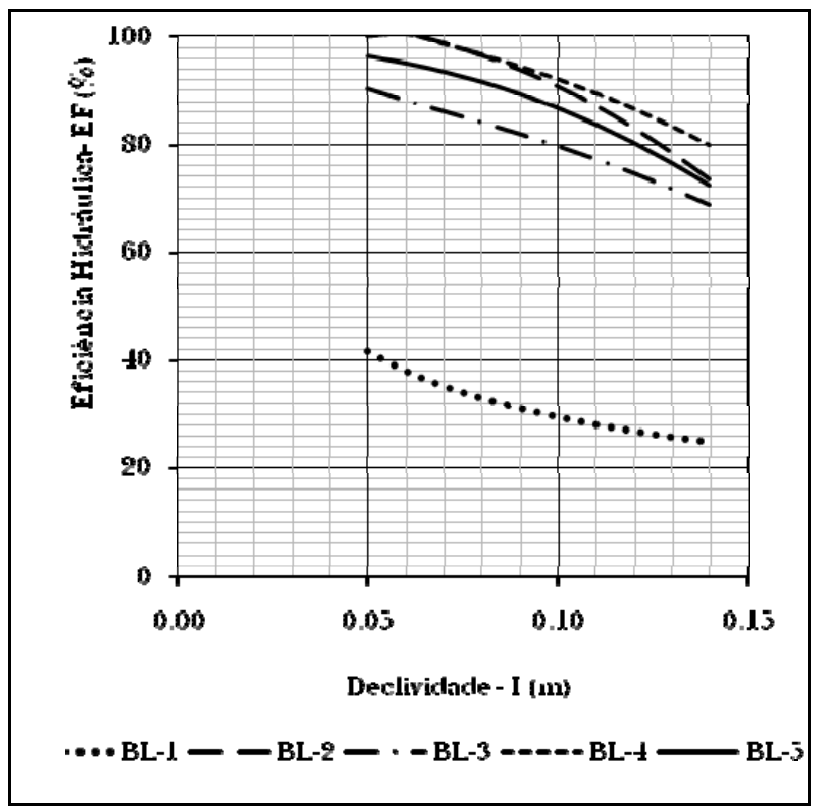

Figura 15 - Eficiência das bocas de lobo, em função das declividades longitudinais, para a faixa de alagamento máxima $(T=1,67 m)$
Nesta figura fica evidente a dependência da eficiência hidráulica das bocas de lobo com a declividade longitudinal da sarjeta, sendo os maiores valores de eficiência obtidos para as menores declividades. A variação dessas eficiências hidráulicas (EF) para os tipos de bocas de lobo ensaiadas estão descritos a seguir:

- $\quad$ BL-1: $25 \%<\mathrm{EF}<42 \%$

- BL-2: $73 \%<\mathrm{EF}<100 \%$

- BL-3: $69 \%<\mathrm{EF}<90 \%$

- BL-4: $80 \%<\mathrm{EF}<100 \%$

- BL-5: $72 \%<\mathrm{EF}<96 \%$

\section{CONGLUSÕES}

Ao longo desse trabalho, o comportamento hidráulico de cinco tipos de bocas de lobo foi pesquisado, objetivando avaliar a capacidade de captação das mesmas, quando inseridas em sarjetas de greide contínuo.

Como síntese global dos resultados obtidos na presente pesquisa, destaca-se:

- As bocas de lobo apresentaram maiores eficiências hidráulicas para as menores declividades longitudinais nas sarjetas.

- A boca de lobo simples, BL-1, dotada de uma abertura na guia somente, apresentou as menores eficiências hidráulicas (entre $25 \%$ e $42 \%$ ).

- A boca de lobo, BL-4, dotada de grelha, abertura na guia e depressão na sarjeta, apresentou as maiores eficiências hidráulicas (entre $80 \%$ e $100 \%$ ).

- A depressão na sarjeta, junto à boca de lobo BL-4 e BL-5, aumentou as eficiências hidráulicas de $3 \%$ a $7 \%$, quando comparada com a BL-2 e BL-3, sem depressão. Contu- 
do, para declividades inferiores a $9 \%$ a depressão da boca de lobo BL-4 não foi eficaz, quando comparada a BL-2.

- A abertura na guia, junto à boca de lobo combinada BL-2 e BL-4, quando comparada, com a boca de lobo BL-3 ou BL-5, respectivamente, aumentou a eficiência hidráulica de $4 \%$ a $8 \%$.

Os testes também indicaram que a equação de Manning produz melhores resultados que a de Izzard para determinação da vazão escoada na sarjeta. Assim, conhecendo-se a vazão na sarjeta, para a faixa de alagamento máxima por meio da equação de Manning (ver equação 9) e a eficiência hidráulica dos diversos tipos de boca de lobo, mostrados na Figura 15, é possível prever as vazões captadas pelas bocas de lobo.

Aplicando esta metodologia à boca de lobo combinada, em sarjeta com depressão (BL-4), para as declividades de $5 \%$ e $8 \%$, obtêm-se as vazões de captação de 85 l/s e 104 l/s, respectivamente. Para as mesmas circunstâncias, o critério utilizado atualmente pela SUDECAP recomenda as vazões de captação de 102 l/s e 128 l/s. Constata-se, portanto, nestes casos, um subdimensionamento do sistema de microdrenagem, em torno de $20 \%$, oriundos, principalmente das diferenças entre os modelos físicos utilizados nas pesquisas da Universidade de Johns Hopkins, fonte utilizada para o estabelecimento do critério e da presente pesquisa.

\section{AGRADECIMENTOS}

Agradecemos ao CNPq - Conselho Nacional de Desenvolvimento Científico e Tecnológico, à CAPES - Coordenação de Aperfeiçoamento de Pessoal de Nível Superior e à FAPEMIG - Fundação de Amparo à Pesquisa do Estado de Minas Gerais pelos apoios financeiros concedidos para o desenvolvimento científico e tecnológico das pesquisas realizadas.

\section{REFERÊNCIAS}

CARDOSO, C. H. A.; GenOVEZ, A. I. B.; GENOVEZ, A. M. "Eficiência de captação de águas pluviais com defletores." in Anais do 10 Congresso da água, 2004, Campinas. São Paulo, 2004.
DAEE/CETESB - Drenagem Urbana: Manual de Projeto. São Paulo: 468p., 1980.

DALFRÉ P. M. O.; GENOVEZ A. I. B. "Eficiência de captação em boca de lobo com depressão e rasgo adicional" in Anais do XXI Congresso latino americano de hidráulica, São Pedro. São Paulo, 2004.

EASTWOOD, W. Theory of overland flow and its application to the design of drain inlet on roads. The Suveyor. 105 Aug. 1946 pp 651-5, citado por SOUZA, P.A., Eficiência Hidráulica da Boca de Lobo. Boletim 3. CTH, São Paulo, 1986.

KRIGUER, E. K.; CONTRERAS, R. C. "Plataforma de pendientes variables para la experimentación de sumideros de aguas Iluviais" in Anais do XXI Congresso latino americano de hidráulica, São Pedro. São Paulo, 2004.

LIMA, J. G. A., COELHO, M. M. L. P. "Avaliação das capacidades das sarjetas e bocas de lobo" in Anais do $23^{\circ}$ Congresso Brasileiro de Engenharia Sanitária e Ambiental, Belo Horizonte, MG, 2007.

LIMA, J. G. A., COELHO, M. M. L. P. "Investigações experimentais sobre as capacidades das bocas de lobo em Greide Contínuo" in Anais do XVII Simpósio Brasileiro de Recursos Hídricos, São Paulo, SP, 2007.

LIMA, J. G. A. Investigações experimentais da eficiência hidráulica em bocas de lobo em greide contínuo. 2007. Dissertação (Mestrado) - Programa de PósGraduação em Saneamento Meio Ambiente e Recursos Hídricos.

LI, W.H.; GEYER, J.C.; BENTON, G.S. "Flow into gutter inlets in a straight gutter without depression, reimpresso de Sewage and Industrial Wastes", v. 23 n. 1, January,1951 e publicado em The Design of Storm-Water Inlets.

LI, W.H.; GEYER, J.C.; SORTEBERG, K.K, Flow into Curb_Openning Inlets, reimpresso Sewage and Industrial Wastes, vol. 23, n. 6 June, 1951 e publicado em The Design of Storm-Water Inlets.

LI, W. H.; GOODELL, B.C.; GEYER, J. C. "Flow Into Deflector Inlets, reimpresso de Sewage and Industrial Wastes", vol. 26, n. 7, July, 1954 e Publicado em The Design of Storm-Water Inlets.

LI, W. H.; GOODELL, B. C.; GEYER, J. C. "Flow Into Depressed Combination Inlets", reimpresso Sewage and Industrial Wastes, vol. 26, n. 8, August, 1954 e Publicado em The Design of Storm-Water Inlets.

RUSSAM, K. The Hidraulic efficiency and Spacing of British Standard Road Gulleys. Water Research Laboratory. Report LR 277, 1969, citado por SOUZA, P.A., Eficiência Hidráulica da Boca de Lobo. Boletim 3. CTH, São Paulo, 1986.

SOUZA, P. A., "Eficiência Hidráulica da Boca de lobo". Boletim 3. Centro Tecnológico de Hidráulica, DAEE, São Paulo, 36 p., 1986. 
SUDECAP. Instrução Técnica para Elaboração de Estudos e Projetos de Drenagem Urbana. Belo Horizonte, 53 p., 2004.

YONG, K,C. Hydraulic Model Investigation of Kerb-Opening Inlets. Water Research Laboratory, The University of New South Wales. Report No 79, Austrália 1965, citado por SOUZA, P.A., Eficiência Hidráulica da Boca de Lobo. Boletim 3. CTH, São Paulo, 1986.

ZWAMBORN, J.A. Stormwater inlet design. In: Annual Municipal Conference, 1966, Juhannesburg, may, 1966, citado por SOUZA, P. A., Eficiência Hidráulica da Boca de Lobo. Boletim 3. CTH, São Paulo, 1986.

\section{Hydraulic Efficiency of Street Inlets in a Straight gutter}

\section{ABSTRACT}

Gutter inlets are important hydraulic structures in urban drainage systems. They are responsible for the intake of water flowing in the gutters. In most Brazilian cities, the design of these structures is based on the results of research with a physical model that is very different from those currently used on our streets. For this reason, the paper presents the results obtained using a scale model that aims at reproducing a short segment of street in continuous slope, equipped with gutter inlets, similar to those adopted by the drainage systems in Belo Horizonte. By running tests with this model it was possible to assign an intake law for each type of gutter inlet tested. The main results may be summarized as follows: the gutter inlets have a higher hydraulic efficiency for smaller longitudinal slopes in the gutter; the simple gutter inlet, which has an opening in the curb only, is least efficient, while the depressed combination inlet has the highest hydraulic efficiency, that can reach the value of $100 \%$ for gutters having a longitudinal slope below 6\%; the contribution of the opening in the curb or the depression, to the depressed combination inlet is small, less than 8\%. The tests also indicated a good fit of the Manning equation to estimate the flow drained into the gutter. A flow intake estimate is proposed based on data on the flow into the gutter and the hydraulic efficiency of the gutter inlet.

Keywords: Drainage, gutter inlet, gutter. 